\title{
Respostas de Freqüência Cardíaca, Consumo de Oxigênio e Sensação Subjetiva ao Esforço em um Exercício de Hidroginástica Executado por Mulheres em Diferentes Situações Com e Sem o Equipamento Aquafins ${ }^{\circledR}$

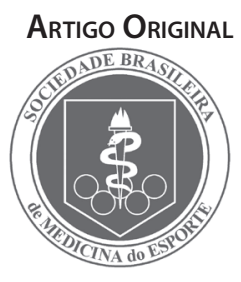

\author{
Heart Rate, Oxygen Consumption and Rating of Perceived Exertion Responses \\ in a Water Aerobic Exercise Performed by Women at Different Situations \\ with and without the Aquafins Apparatus
}

Stephanie Santana Pinto Cristine Lima Alberton Paulo André Poli de Figueiredo Carlos Leandro Tiggemann Luiz Fernando Martins Kruel

Grupo de Pesquisa em Atividades Aquáticas e Terrestres, Escola de Educação Física

Universidade Federal do Rio Grande do Sul.

Porto Alegre, Rio Grande do Sul, Brasil.

Endereço para correspondência: Stephanie Santana Pinto Laboratório de Pesquisa do

Exercício, sala 208

Rua Felizardo, 750 - Jardim

Botânico

90690-200 - Porto Alegre, RS, Brasil E-mail: tetisantana@yahoo.com.br

Submetido em 26/01/2007 Versão final recebida em 11/02/2008 Aceito em 22/02/2008

\section{RESUMO}

As aulas de hidroginástica estão cada vez mais diversificadas com o uso de materiais apropriados ao meio aquático. Entretanto, poucos estudos verificaram a influência da utilização de tais materiais nas respostas cardiorrespiratórias. O objetivo foi analisar as respostas de freqüência cardíaca, consumo de oxigênio e sensação subjetiva ao esforço em mulheres durante a execução de um exercício de hidroginástica em diferentes situações com e sem o equipamento resistivo Aquafins ${ }^{\circledR}$. Onze mulheres realizaram o exercício deslize frontal com a flexão e extensão horizontal de ombros em quatro situações: sem equipamento resistivo (S-FINS), com Aquafins ${ }^{\circledR}$ nos membros inferiores (FINS-MIs), com Aquafins ${ }^{\circledR}$ nos membros superiores (FINS-MSs) e com Aquafins ${ }^{\circledR}$ nos membros superiores e inferiores (FINS-MIs/MSs). Em todas as situações foi verificado a $\mathrm{FC}_{1} \mathrm{o} \mathrm{VO}_{2}$ e a SSE. Utilizou-se ANOVA para medidas repetidas, com post-hoc de Bonferroni $(p<0,05)$. A FC foi significativamente mais elevada nas situações FINS-MIs/MSs (159 \pm 12bpm) e FINS-MIs (147 \pm 18bpm), comparando-as com as demais situações. No entanto, a situação FINS-MIs apresentou FC similar à FINS-MSs (148 \pm 16 bpm). Por sua vez, as três situações com o Aquafins ${ }^{\circledR}$ foram diferentes da situação S-FINS (131 $\pm 14 \mathrm{bpm}$ ). Para o VO houve diferença significativa entre a situação FINS-MIs/MSs $\left(22,77 \pm 3,58 \mathrm{ml}^{\mathrm{kg}} \mathrm{kg}^{-1} \cdot \mathrm{min}^{-1}\right)$, comparando-a com as demais. As situações FINS-MSs (19,67 $\left.\pm 4,29 \mathrm{ml} . \mathrm{kg}^{-1} \cdot \mathrm{min}^{-1}\right)$ e FINS-Mls $\left(20,38 \pm 3,99 \mathrm{ml} . \mathrm{kg}^{-1} \cdot \mathrm{min}^{-1}\right)$ apresentaram

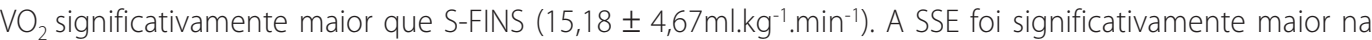
situação FINS-MIs/MSs (16 \pm 1,55), comparando-a com FINS-MIs (14 \pm 0,9) e S-FINS (13 \pm 1,29), entretanto, não foi diferente da FINS-MSs $(14 \pm 1,57)$. Portanto, a utilização de equipamentos que promovam resistência ao movimento na hidroginástica é indicada para melhorias no sistema cardiorrespiratório.

Palavras-chave: freqüência cardíaca, consumo de oxigênio, esforço percebido, equipamento resistivo.

\section{ABSTRACT}

Aquatic exercise sessions are becoming increasingly diversified due to the use of apparatus appropriate to aquatic environment. However, few studies analyzed the influence of the use of such apparatus in the cardiorespiratory responses. The purpose was to analyze the heart rate, oxygen consumption and the rating of perceived exertion of effort in women during an aquatic exercise performed in different situations with and without resistive equipment, Aquafins ${ }^{\mathrm{TM}}$. Eleven women performed the cross country skiing exercise with horizontal shoulder flexion and extension in four situations: without resistive equipment (NO-FINS), with Aquafins ${ }^{\mathrm{TM}}$ on the lower limbs (FINS-LLs), with Aquafins ${ }^{\mathrm{TM}}$ on the upper limbs (FINS-ULs) and with Aquafins ${ }^{\mathrm{TM}}$ on the lower and upper limbs (FINS-LLS/ULS). In each situation $\mathrm{HR}, V_{2}$ and SSE were verified. For data analysis, ANOVA for repeated measures were used, with the Bonferroni post-hoc test $(p<0.05)$. When compared to the other situations, HR was significantly higher with the FINS-LLs/ULs (159 \pm 12 bpm) and FINS-LLs (147 \pm 18 bpm). However, the FINS-LLs situation exhibited a similar HR to that of FINS-ULs (148 \pm 16 bpm). In turn, the three situations with Aquafins $^{\mathrm{TM}}$ were different from the NO-FINS (131 $\left.\pm 14 \mathrm{bpm}\right)$ situation. In the case of $\mathrm{VO}_{2}$, there was a significant difference between the FINS-LLS/ULS $\left(22.77 \pm 3.58 \mathrm{ml}^{\mathrm{kg}} \mathrm{kg}^{-1} \cdot \mathrm{min}^{-1}\right)$ situation and the other situations. The FINS-ULS $\left(19.67 \pm 4.29 \mathrm{ml} . \mathrm{kg}^{-1} \cdot \mathrm{min}^{-1}\right)$ and FINS-LLs $\left(20.38 \pm 3.99 \mathrm{ml} \cdot \mathrm{kg}^{-1} \cdot \mathrm{min}^{-1}\right)$ situations exhibited significantly higher $\mathrm{VO}_{2}$ levels than NO-FINS $\left(15.18 \pm 4.67 \mathrm{ml}^{\mathrm{kg}}{ }^{-1} \cdot \mathrm{min}^{-1}\right)$. The RPE was significantly greater in the FINS-LLS/ULs (16 \pm 1.55$)$ situation when compared to FINS-LLs (14 \pm 0.9$)$ and NO-FINS (13 \pm 1.29$)$, though there was no difference from the FINS-ULS (14 \pm 1.57$)$ situation. Therefore, the use of equipments that promote resistence to the movement in aquatic exercise is recommended to improvement in the cardiorespiratory system.

Keywords: heart rate, oxygen consumption, perceived exertion, resistive apparatus. 


\section{INTRODUÇÃO}

Os exercícios físicos realizados no meio aquático na posição vertical, como a hidroginástica, estão sendo cada vez mais indicados devido aos seus diversos benefícios à saúde ${ }^{(1-2)}$. Essa modalidade pode promover melhoria nos diversos componentes da aptidão física, como força ${ }^{(3-6)}$, flexibilidade ${ }^{(5,7)}$, composição corporal ${ }^{(5)}$ e condicionamento cardiorrespiratório ${ }^{(5,8-9)}$, quando prescrita adequadamente para tais objetivos. Além disso, exercícios realizados na água apresentam reduzido impacto comparado com o meio terrestre ${ }^{(2,10)}$ e comportamento de freqüência cardíaca e pressão arterial mais baixos ${ }^{(11-12)}$.

Os benefícios que um programa de exercícios realizado no meio aquático proporciona no condicionamento geral do corpo estão relacionados com as propriedades físicas da água. Podemos destacar, dentre essas propriedades, a resistência ao avanço $(R)$, que pode ser expressa como $R=0,5 * \rho * A * V^{2} * C d$, onde, $\rho$ é a densidade do fluido, $A$ é a área de superfície projetada, $v$ é a velocidade do movimento e Cd é o coeficiente de arrasto $^{(13)}$. Nas modalidades realizadas no meio aquático, podem-se utilizar exercícios que proporcionam maior resistência ao avanço, com conseqüente incremento de intensidade dos mesmos, através do aumento da velocidade de execução e/ou através da alteração da área projetada, que pode ser modificada pela utilização do corpo de diferentes formas na água e também pela execução de exercícios com o uso de equipamentos que promovam resistência ao movimento ${ }^{(14)}$.

As aulas de hidroginástica estão cada vez mais diversificadas com o uso de diferentes materiais apropriados ao meio aquático. Entretanto, existem poucos estudos verificando a influência da utilização de tais materiais nos parâmetros fisiológicos. No que se refere à utilização de equipamentos resistivos, alguns estudos analisaram os efeitos de treinamento de força no meio aquático com grupos treinando com e sem equipamento(6). Os resultados mostraram que houve incremento de força similar em ambos os grupos. Resultados semelhantes são encontrados com estudos analisando a atividade eletromiográfica de determinados músculos em movimentos específicos com e sem o equipamento resistivo, demonstrando também uma mesma ativação muscular para os movimentos executados com e sem o equipamento resistivo na velocidade máxima de execução ${ }^{(15)}$.

Na literatura pesquisada foi encontrado apenas um estudo relacionando dois exercícios de hidroginástica executados com e sem o uso de diferentes equipamentos resistivos nos membros inferiores e suas respectivas respostas cardiorrespiratórias ${ }^{(16)}$. Os resultados mostraram que houve aumento significativo no consumo de oxigênio $\left(\mathrm{VO}_{2}\right)$ com o uso de ambos os equipamentos resistivos para um dos exercícios analisados. Já o comportamento da freqüência cardíaca ( $F C$ ) foi significativamente mais elevado com o uso dos equipamentos resistivos para os dois exercícios comparados com a situação sem equipamento. Ainda é importante salientar que, ao compararem a utilização do equipamento Aquafins ${ }^{\circledR}$ e Aqualogger ${ }^{\circledR}$, houve diferença entre eles apenas para a FC em ambos os exercícios. Entretanto, nesse estudo de Pinto et al. ${ }^{(16)}$ não foram analisados os efeitos da utilização de equipamentos nos membros superiores nas respostas cardiorrespiratórias quando comparados aos membros inferiores e também não foi verificada a sensação subjetiva ao esforço.

Com relação à execução de exercícios aeróbicos utilizando movimentos combinados de membros superiores e inferiores, a literatura demonstra não existir uma relação linear entre a $\mathrm{FC} \mathrm{e} \mathrm{o} \mathrm{VO}_{2}$, tanto no meio aquático como no terrestre ${ }^{(1,17)}$. Isso pode ser explicado pelo fato de o exercício com os membros superiores produzir aumento da freqüência cardíaca através de ativação simpática no sistema cardiovascular, enquanto que o $\mathrm{VO}_{2}$ não sofre a mesma alteração durante a execução do mesmo exercício em uma mesma intensidade ${ }^{(18)}$. Partindo desse pressuposto, a realização de exercícios aquáticos com a utilização de equipamentos nos membros superiores, nos membros inferiores ou em ambos poderia resultar em respostas distintas.

Portanto, o objetivo do presente estudo foi analisar as alterações nas respostas de freqüência cardíaca $(\mathrm{FC})$, consumo de oxigênio $\left(\mathrm{VO}_{2}\right)$ e sensação subjetiva ao esforço (SSE) em mulheres durante a execução de um exercício de hidroginástica em diferentes situações com e sem o equipamento resistivo Aquafins ${ }^{\circledR}$. A hipótese dos autores é de que as variáveis analisadas apresentarão comportamento diferente entre as quatro situações de utilização ou não de equipamento.

\section{METODOLOGIA}

A amostra deste estudo foi composta por 11 mulheres jovens ambientadas ao meio líquido, com idade entre 19 e 26 anos. Todas realizavam a prática regular de aulas de hidroginástica de duas a três vezes por semana. A seleção da amostra foi realizada através de convite verbal para os alunos do Centro Natatório da Escola de Educação Física da Universidade Federal do Rio Grande do Sul. As voluntárias assinaram um termo de consentimento informado, no qual constavam todas as informações pertinentes ao estudo. Como critérios de inclusão foram adotados: isenção de problemas ostearticulares, não estar tomando medicação, ser familiarizada com o meio líquido e praticar hidroginástica por no mínimo um ano.

Na primeira sessão de avaliação, foram obtidas as medidas de massa corporal e estatura, em uma balança de alavanca Filizola, com estadiômetro acoplado, e um teste de esforço máximo, em uma esteira 10200 ATL, da marca Inbramed (Porto Alegre, Brasil). O protocolo usado para determinação do $\mathrm{VO}_{2}$ de pico $\left(\mathrm{VO}_{2 \text { pico }}\right)$ foi em rampa, com velocidade inicial de $5 \mathrm{~km} \cdot \mathrm{h}^{-1}$ e incrementos de $1 \mathrm{~km} \cdot \mathrm{h}^{-1}$ a cada minuto, com elevação fixa em 1\%.

A seguir foi realizada uma sessão de testes aquáticos no Centro Natatório da Escola de Educação Física da Universidade Federal do Rio Grande do Sul. Nessa sessão, os indivíduos realizaram o exercício deslize frontal combinado com a flexão e extensão horizontal de ombros (DF-FE), conforme figura 1. Esse exercício foi realizado na cadência de 60bpm, que foi reproduzida por um metrônomo modelo Matrix MR-500 da marca Quartz, durante quatro minutos, nas seguintes situações (figura 2): sem o uso de equipamento resistivo (S-FINS), com o uso do equipamento resistivo Aquafins ${ }^{\circledR}$ nos membros superiores (FINS-MSs), com o uso do equipamento resistivo Aquafins ${ }^{\circledR}$ nos membros inferiores (FINS-MIs) e com o uso do equipamento resisti-

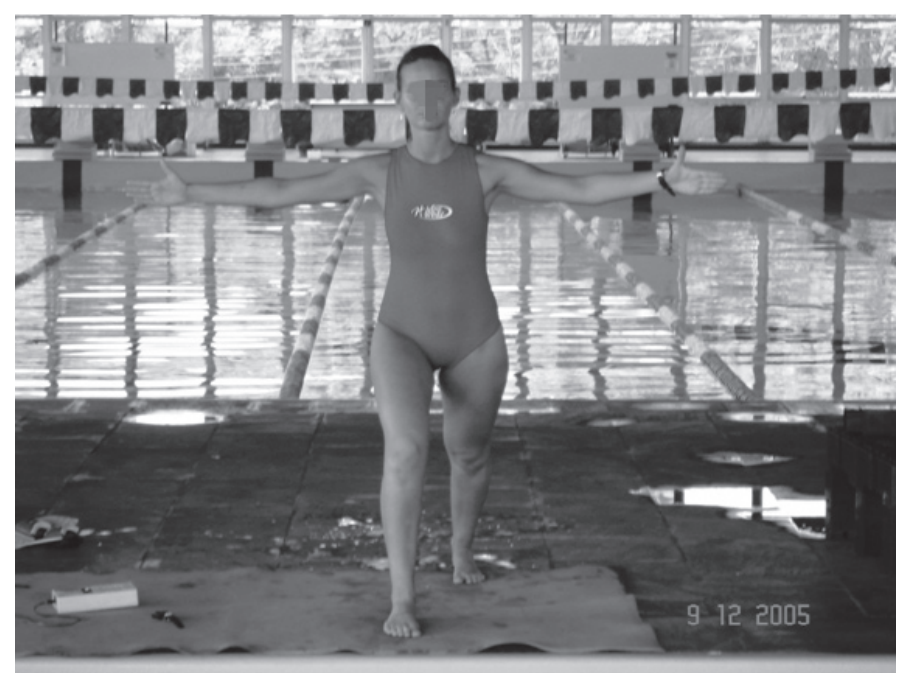

Figura 1. Exercício deslize frontal combinado com a flexão e extensão horizontal de ombros. 


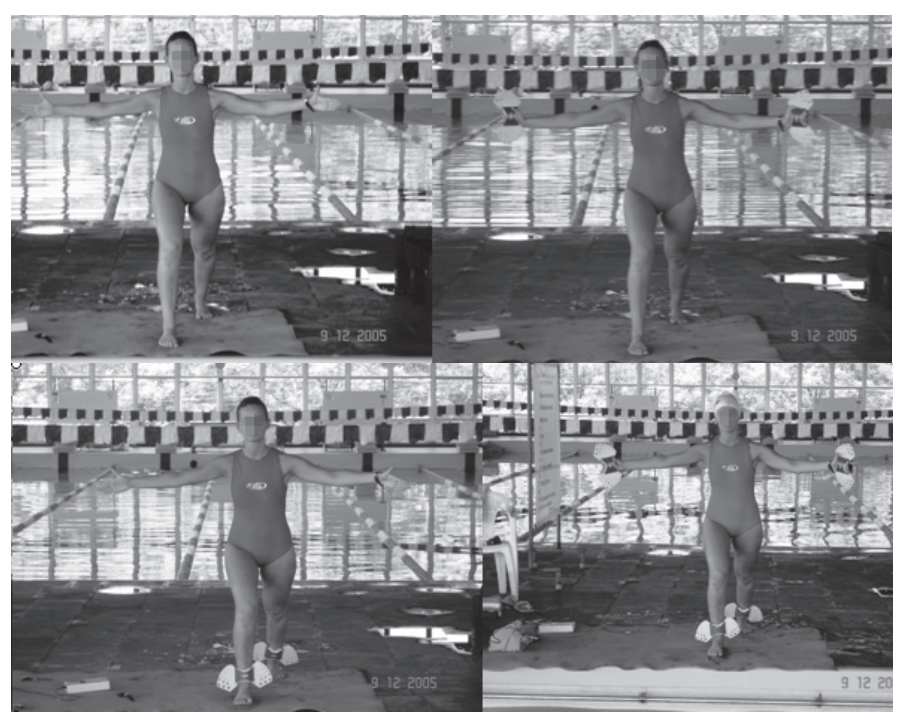

Figura 2. Execução do exercício deslize frontal combinado com a flexão e extensão horizontal de ombros nas diferentes situações: sem equipamento resistivo (S-FINS), com equipamento Aquafins ${ }^{\circledR}$ nos membros superiores (FINS-MSs), com equipamento Aquafins ${ }^{\circledR}$ nos membros inferiores (FINS-MIs) e com o equipamento Aquafins ${ }^{\circledR}$ nos membros superiores e inferiores (FINS-MIs/MSs).

vo Aquafins ${ }^{\circledR}$ nos membros superiores e inferiores simultaneamente (FINS-MSs/MIs). O equipamento Aquafins ${ }^{\circledR}$ foi escolhido pelo fato de proporcionar grande resistência na água e ser de fácil utilização, devido ao seu material e design que permitem seu uso nos membros superiores e inferiores.

As situações foram randomizadas através do método do quadrado latino ${ }^{(19)}$ e respeitaram intervalos de 20 minutos. O equipamento Aquafins ${ }^{\circledR}$ nos membros inferiores foi cuidadosamente localizado $3 \mathrm{~cm}$ acima do maléolo medial, com as abas posicionadas no plano frontal, proporcionando resistência aos movimentos ântero-posteriores durante o exercício deslize frontal. Já nos membros superiores, os indivíduos seguravam o equipamento na palma das mãos com as abas posicionadas com intuito de proporcionar resistência aos movimentos contralaterais durante o exercício de flexão e extensão horizontal de ombros.

Em ambas as sessões de testes, para a verificação da freqüência cardíaca (FC) foi utilizado um freqüencímetro S610, da marca Polar (Kajaani, Finlândia) e, para a verificação do consumo de oxigênio $\left(\mathrm{VO}_{2}\right)$, foi utilizado um analisador de gases portátil KB1-C, da marca Aerosport (Ann Arbor, EUA) ${ }^{(16)}$. Antes do início do procedimento da coleta de dados foi realizada calibração com gases específicos; calibração automática também foi realizada antes de cada situação. Os critérios estabelecidos para a coleta desses dados foram: alimentar-se no período de 3 a 4 h antes do início da sessão de testes, sem a ingestão de estimulantes, e evitar a prática de atividades físicas intensas durante as últimas $24 \mathrm{~h}^{(20)}$. Os valores dessas variáveis foram obtidos a cada 20 segundos.

Para análise desses dados, utilizou-se a média dos valores coletados entre o terceiro e o quarto minuto da execução do exercício em cada situação, correspondente à $\mathrm{FC}$ e ao $\mathrm{VO}_{2}$ em steady state ${ }^{(21)}$. Além disso, foi calculado o percentual do $\mathrm{VO}_{2 \text { pico }}\left(\% \mathrm{VO}_{2 \text { pico }}\right)$ nas diferentes situações através do valor de referência do $\mathrm{VO}_{2 \text { pico }}$ obtido no teste máximo.

Por fim, a verificação da sensação subjetiva ao esforço (SSE) geral, que pode ser definida como um esforço percebido que está intimamente relacionado com as respostas do sistema musculoesquelético, cardiovascular e pulmonar, foi realizada através da escala RPE de Borg. Essa escala apresenta os níveis de 6 a 20, sendo que 6 significa "sem nenhum esforço"e 20, "máximo esforço". A SSE local, dividida em mem- bros superiores e inferiores, corresponde à percepção de esforço nos grupos musculares solicitados no exercício executado, e também foi verificada através da escala citada anteriormente. Os sujeitos indicavam sua estimativa de esforço, tanto geral como local, ao final do exercício em cada uma das situações.

Os testes foram realizados em uma piscina com profundidade entre 1,10 e 1,40m. Isso permitia que a profundidade de imersão ficasse entre o processo xifóide e ombros. A temperatura da água foi mantida entre 32 e $33^{\circ} \mathrm{C}$. Essas variáveis foram controladas para evitar sua influência nas respostas fisiológicas ${ }^{(22-23)}$.

Para a análise dos dados coletados foi utilizada estatística descritiva (média \pm desvio-padrão). Verificou-se a normalidade dos dados através do teste de Shapiro-Wilk. Para análise das variáveis dependentes entre as diferentes situações foi utilizado ANOVA para medidas repetidas, com o teste post-hoc de Bonferroni. O nível de significância adotado neste estudo foi $p<0,05$. Todos os dados foram processados no programa estatístico SPSS versão 13.0.

\section{RESULTADOS}

Para a amostra do presente estudo foram avaliadas 11 mulheres jovens voluntárias, ambientadas ao meio líquido, com as características apresentadas na tabela 1.

Tabela 1. Caracterização da amostra com valores de idade, massa, estatura e consumo de oxigênio de pico $\left(\mathrm{VO}_{2 \text { pico }}\right)$ como média e desvio-padrão (DP).

\begin{tabular}{c|c|c}
\hline $\mathbf{N}=\mathbf{1 1}$ & MÉDIA & DP \\
\hline Idade (anos) & 22,0 & $\pm 2,0$ \\
\hline Massa $(\mathrm{kg})$ & 58,3 & $\pm 6,5$ \\
\hline Estatura $(\mathrm{cm})$ & 163,4 & $\pm 7,0$ \\
\hline $\mathrm{VO}_{2 \text { pico }}\left(\mathrm{ml}^{\mathrm{kg}} \mathrm{kg}^{-1} \cdot \mathrm{min}^{-1}\right)$ & 41,1 & $\pm 2,8$ \\
\hline
\end{tabular}

Os resultados da $\mathrm{FC}$, do $\mathrm{VO}_{2}$ e do $\% \mathrm{VO}_{2 \text { pico }}$ entre as diferentes situações, no exercício DF-FE são apresentados na tabela 2. O comportamento da FC foi significativamente mais elevado nas situações FINS-MIs/MSs e FINS-MIs, comparando-as com as demais situações. A situação FINS-Mls também apresentou comportamento de FC semeIhante ao da FINS-MSs. Por sua vez, as três situações com o Aquafins ${ }^{\circledR}$ foram diferentes da situação S-FINS. Para o $\mathrm{VO}_{2}$ e \% $\mathrm{VO}_{2 \text { pico }}$ houve diferença significativa entre a situação FINS-MIs/MSs, comparando-a com as demais. As situações FINS-MSs e FINS-MIs apresentaram comportamento semelhante de $\mathrm{VO}_{2}$ e \% $\mathrm{VO}_{2 \text { picor }}$ que foi significativamente mais elevado que S-FINS.

Tabela 2. Valores de freqüência cardíaca ( $\mathrm{FC})$, consumo de oxigênio $\left(\mathrm{VO}_{2}\right)$ e percentual do consumo de oxigênio de pico $\left(\% \mathrm{VO}_{2 \text { pico }}\right)$ nas situações sem equipamento resistivo (S-FINS), com equipamento Aquafins ${ }^{\circledR}$ nos membros superiores (FINS-MSs), com equipamento Aquafins ${ }^{\circledR}$ nos membros inferiores (FINS-MIs) e com o equipamento Aquafins ${ }^{\circledR}$ nos membros superiores e inferiores (FINS-MIs/MSs)

\begin{tabular}{c|c|c|c|c|c|c}
\hline \multirow{2}{*}{ Situação } & \multicolumn{2}{|c|}{$\mathrm{FC}(\mathrm{bpm})$} & $\mathrm{VO}_{2}\left(\mathrm{ml}^{\mathrm{k}} \mathrm{kg}^{-1} \cdot \mathrm{min}^{-1}\right)$ & \multicolumn{2}{c}{$\% \mathrm{VO}_{2 \text { picc }}(\%)$} \\
\cline { 2 - 7 } & Média & $\mathrm{DP}$ & Média & DP & Média & DP \\
\hline S-FINS & $131^{\mathrm{a}}$ & \pm 14 & $15,18^{\mathrm{a}}$ & $\pm 4,67$ & $36,95^{\mathrm{a}}$ & $\pm 11,35$ \\
\hline FINS-MSS & $148^{\mathrm{b}}$ & \pm 16 & $19,67^{\mathrm{b}}$ & $\pm 4,29$ & $47,97^{\mathrm{b}}$ & $\pm 10,28$ \\
\hline FINS-MIs & $147^{\mathrm{bc}}$ & \pm 18 & $20,38^{\mathrm{b}}$ & $\pm 3,99$ & $49,77^{\mathrm{b}}$ & $\pm 9,80$ \\
\hline FINS-MIs/MSs & $159^{\mathrm{c}}$ & \pm 12 & $22,77^{\mathrm{c}}$ & $\pm 3,58$ & $55,48^{\mathrm{c}}$ & $\pm 7,79$ \\
\hline
\end{tabular}

Letras diferentes representam diferenças significativas $(p<0,05)$ entre as situações. 
Os resultados da SSE geral entre as diferentes situações são apresentados na figura 3. A SSE geral foi significativamente maior na situação FINS-MIs/MSs, comparando-a com a FINS-MIs e S-FINS, entretanto, não foi diferente da FINS-MSs. Já a FINS-MSs também foi igual à FINS-MIs e à S-FINS. Por fim, a situação FINS-MIs foi igual à da S-FINS.

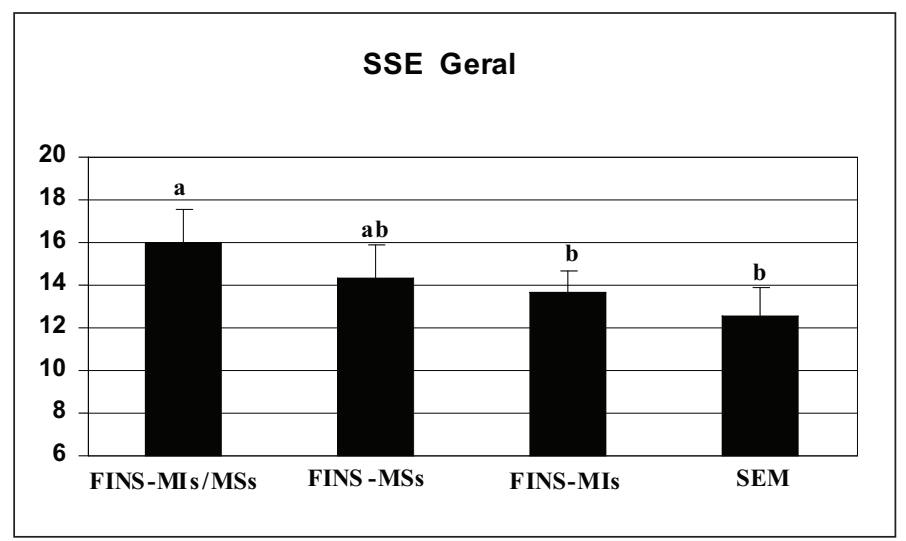

Figura 3. Sensação subjetiva ao esforço geral (SSE) nas situações com o equipamento Aquafins ${ }^{\circledR}$ nos membros superiores e inferiores (FINS-MIs/MSs), com equipamento Aquafins ${ }^{\circledR}$ nos membros superiores (FINS-MSs), com equipamento Aquafins ${ }^{\circledR}$ nos membros inferiores (FINS-MIs) e sem equipamento resistivo (S-FINS). Obs.: letras diferentes representam diferenças significativas $(p<0,05)$ entre as situações.

Os resultados da SSE local de MIs e MSs entre as diferentes situações são apresentados nas figuras 4 e 5, respectivamente. A SSE local de MIs foi significativamente maior nas situações FINS-MIs/MSs e FINS-Mls, comparando-as com FINS-MSs e à S-FINS. Para a SSE local de MSs encontraram-se valores maiores e significativos nas situações FINS-MIs/MSs e FINS-MSs, comparando-as com as demais.

\section{DISCUSSÃO}

Conforme os resultados do presente estudo, nossa hipótese foi parcialmente aceita, visto que o exercício executado com o uso do equipamento resistivo Aquafins ${ }^{\circledR}$ em ambos os membros apresentou maiores respostas de $\mathrm{FC}_{1} \mathrm{VO}_{2}$ e SSE geral e local, e o exercício sem o uso de equipamento obteve as menores respostas nessas variáveis. Porém, o uso de tal equipamento somente nos membros superiores ou inferiores proporcionou respostas similares para a $\mathrm{FC}$ e o $\mathrm{VO}_{2}$. Ainda, embora não tenha sido encontrada diferença significativa para

\section{SSE Local MIs}

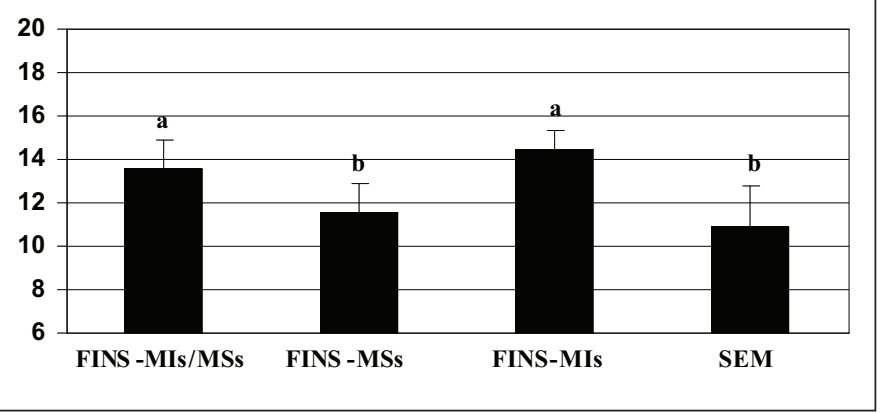

Figura 4. Sensação subjetiva ao esforço local de membros inferiores (SSE MIs) nas situações com o equipamento Aquafins ${ }^{\circledR}$ nos membros superiores e inferiores (FINSMls/MSs), com equipamento Aquafins ${ }^{\circledR}$ nos membros superiores (FINS-MSs), com equipamento Aquafins ${ }^{\circledR}$ nos membros inferiores (FINS-MIs) e sem equipamento resistivo (S-FINS). Obs.: letras diferentes representam diferenças significativas $(p<0,05)$ entre as situações.

\section{SSE Local MSs}

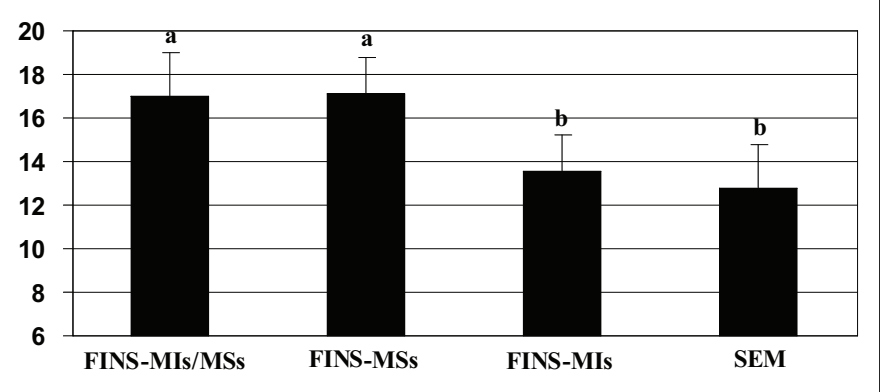

Figura 5. Sensação subjetiva ao esforço local de membros superiores (SSE MSs) nas situações com o equipamento Aquafins ${ }^{\circledR}$ nos membros superiores e inferiores (FINS-MIs/MSs), com equipamento Aquafins ${ }^{\circledR}$ nos membros superiores (FINS-MSs), com equipamento Aquafins ${ }^{\circledR}$ nos membros inferiores (FINS-MIs) e sem equipamento resistivo (S-FINS). Obs.: letras diferentes representam diferenças significativas $(p<0,05)$ entre as situações.

a SSE geral entre essas duas situações, houve tendência de o uso do equipamento nos membros superiores apresentar maior resposta de SSE.

O uso do equipamento resistivo nas diferentes situações aumentou a intensidade do exercício pelo aumento da área projetada que, conseqüentemente, aumentou a resistência ao avanço(4), maximizando as respostas cardiorrespiratórias. Embora o Aquafins ${ }^{\circledR}$ apresente uma área projetada pequena em relação à área do segmento perna ou braço, provavelmente seu coeficiente de arrasto associado ao aumento de área projetada é suficiente para influenciar a magnitude da resistência oferecida no meio aquático. Esses dados corroboram os resultados de outros estudos que também encontraram aumento nas respostas de $\mathrm{FC}$ e $\mathrm{VO}_{2}$ conforme o aumento da intensidade de execução dos exercícios $^{(14,16,24-25)}$.

No caso do estudo de Pinto et al.(16), essa intensidade foi alterada pela escolha de diferentes exercícios e também pela execução dos mesmos com dois tipos de equipamentos resistivos (Aquafins ${ }^{\circledR}$ e Aqua$\left.\operatorname{logger}^{\circledR}\right)$ nos membros inferiores, aumentando a resistência ao avanço no meio aquático. Os resultados mostraram que houve incremento significativo nas respostas de $\mathrm{VO}_{2}$ com o uso do equipamento resistivo apenas no exercício deslize frontal. Já as respostas da mesma variável no exercício deslize lateral não mostraram aumento significativo com o uso de tais equipamentos. Isso provavelmente pode ser explicado pelo fato de o exercício deslize frontal apresentar maior amplitude de movimento e também maior massa muscular envolvida na execução do mesmo em comparação com o exercício deslize lateral.

No presente estudo analisou-se apenas um exercício, portanto, o grupo muscular envolvido na sua execução, provavelmente, sempre foi o mesmo. A intensidade deste estudo foi modificada pela execução do exercício utilizado em diferentes situações com o equipamento resistivo Aquafins $^{\circledR}$, proporcionando, assim, um comportamento distinto entre as variáveis do presente estudo.

Observou-se aumento no $\mathrm{VO}_{2}$ e na $\mathrm{FC}$ com o uso do equipamento Aquafins ${ }^{\circledR}$ nas diferentes situações; houve maior resposta dessa variável quando o exercício foi realizado com o equipamento resistivo em ambos os membros. O uso de tal equipamento tanto nos membros superiores como nos membros inferiores aumentou a ativação muscular, recrutando maior número de unidades motoras durante a execução do exercício ${ }^{(26)}$, ocasionando maior gasto energético. Embora menor massa muscular seja envolvida no exercício de membros superiores 
em comparação com membros inferiores, encontraram-se respostas similares de $\mathrm{VO}_{2}$ e $\mathrm{FC}$ em ambas as situações, provavelmente porque a carga relativa do equipamento foi maior quando utilizado nos membros superiores, proporcionando, assim, intensidade semelhante de esforço.

A sensação subjetiva ao esforço pode ser definida como a tensão relativa que ocorre nos sistemas musculoesquelético, cardiovascular e pulmonar, durante o exercício físico ${ }^{(27)}$. Nos resultados do presente estudo pode-se afirmar que houve aumento nas respostas de SSE geral quando o exercício foi realizado com o equipamento Aquafins ${ }^{\circledR} \mathrm{em}$ ambos os membros e apenas nos membros superiores comparando com as outras situações. Tais respostas parecem estar mais interligadas com o sistema musculoesquelético do que com o sistema cardiorrespiratório. Como citado anteriormente, a musculatura solicitada durante o exercício com o equipamento nos membros superiores é menor em comparação com o uso do mesmo nos membros inferiores. Isso possivelmente faz com que exista maior recrutamento de unidades motoras para os membros superiores, em valores relativos, em comparação com as outras situações sem o equipamento nos membros superiores, aumentando os índices de percepção de esforço durante a execução do exercício ${ }^{(26,28)}$. Em relação à SSE local, pode-se dizer que houve aumento nos índices de percepção de esforço quando o equipamento resistivo foi utilizado nos membros superiores e/ou inferiores, o que corrobora outros estudos que também verificaram a SSE local de membros superiores e inferiores separadamente no meio aquático(29).

Sugere-se que futuras pesquisas sejam realizadas com indivíduos do sexo masculino e com faixa etária mais abrangente. Além disso, no presente estudo analisaram-se as respostas de apenas um exercício nas diferentes situações, limitando, por sua vez, os dados para uma sessão de hidroginástica, na qual se utiliza um repertório de diferentes exercícios no meio aquático.

\section{CONCLUSÃO}

Pode-se concluir que a utilização do equipamento Aquafins ${ }^{\circledR}$ nas diferentes situações aumentou as respostas cardiorrespiratórias e a sensação subjetiva ao esforço para uma cadência submáxima de execução. A situação que apresentou maior aumento de todas as variáveis analisadas no estudo foi a de executar o exercício com o Aquafins ${ }^{\circledR} \mathrm{em}$ ambos os membros. Além disso, independente do posicionamento do equipamento utilizado, membros superiores ou membros inferiores, as respostas de $\mathrm{FC}_{1} \mathrm{VO}_{2}$ e SSE foram semelhantes. Porém, é importante salientar que a utilização do equipamento resistivo nos membros superiores faz com que o indivíduo perceba o exercício mais intenso do que ele realmente é, de acordo com as suas respostas cardiorrespiratórias, visto que essa situação não apresentou diferenças significativas na SSE comparando-a com a situação de utilização do equipamento em ambos os membros.

\section{AGRADECIMENTOS}

Agradecemos aos integrantes do Grupo de Pesquisa em Atividades Aquáticas e Terrestres (GPAT) pelas correções e críticas construtivas.

Todos os autores declararam não haver qualquer potencial conflito de interesses referente a este artigo.

\section{REFERÊNCIAS BIBLIOGRÁFICAS}

1. Eckerson J, Anderson T. Physiological response to water aerobics. J Sports Med Phys Fitness 1992;32:255-61.

2. Kruel LFM. Alterações fisiológicas e biomecânicas em indivíduos praticando exercícios de hidroginástica dentro e fora d'áqua. Santa Maria, 2000. Tese de Doutorado. Universidade Federal de Santa Maria.

3. Müller FG. A treinabilidade da força muscular em idosas praticantes de hidroginástica. Florianópolis, 2002. Dissertação de Mestrado. Universidade do Estado de Santa Catarina.

4. Pöyhönen T, Sipilä S, Keskinen KL, Hautala A, Savolainen J, Mälkiä E. Effects of aquatic resistance training on neuromuscular performance in healthy women. Med Sci Sports Exerc 2002;34:2103-9.

5. Takeshima N, Rogers ME, Watanabe WF, Brechue WF, Okada A, Yamada T, et al. Water-based exercise improves health-related aspects of fitness in older women. Med Sci Sports Exerc 2002;33:544-51.

6. Barella RE, Muller FG, Severo CR, Cardoso AS, Figueiredo PAP, Brentano MA, et al. Efeitos de um treinamento de força aplicado em mulheres praticantes de hidroginástica. Rev Bras Fisiol Exerc 2004;3:136

7. Alves RV, Mota J, Costa MC, Alves JGB. Physical fitness and elderly health effects of hydrogymnastics. Rev Bras Med Esporte 2004;10:38-43.

8. Avellini BA, Shapiro Y, Pandolf KB. Cardio-respiratory physical training in water and on Land. Eur J Appl Physiol 1983;50:255-63

9. Taunton JE, Rhodes EC, Wolski LA, Donelly M, Warren J, Elliot J, et al. Effect of land-based and waterbased fitness programs on the cardiovascular fitness, strenght and flexibility of woman aged 65-75 years. Gerontology 1996;42:204-10

10. Miyoshi T, Shirota T, Yamamoto S, Nakazawa K, Akai M. Effect of the walking speed to the lower limb joint angular displacements, joint moments and ground reaction forces during walking in water Disabil Rehabil 2004:26:724-32

11. Nakanishi Y, Kimura T, Yoko Y. Maximal responses to deep water running at termoneutral temperature J Physiol Anthropol Appl Human Sci 1999;18:31-5.

12. Srámek P, Simecková M, Janski L, Savlíková J, Vybíral S. Human Physiological responses to immersion into water of different temperatures. Eur J Appl Physiol 2000;81:436-42

13. Pöyhönen T, Keskinen KL, Hautala A, Mälkiä E. Determination of hydrodinamic drag forces and drag coefficients on human leg/foot model during knee exercise. Clin Biomech 2000;15:256-60.

14. Alberton CL, Coertjens M, Figueiredo PAP, Kruel LFM. Behavior of oxygen uptake in water exercises performed at different cadences in ad out of water. Med Sci Sports Exerc 2005:37:5103.
15. Pöyhönen T, Kyrolainen H, Keskinen $K L$, Hautala A, Savolainen J, Mälkiä E. Electromyographic and kinematic analysis of therapeutic knee exercises under water. Clin Biomech 2001;16:496-504.

16. Pinto SS, Alberton CL, Becker ME, Olkoski MM, Kruel LFM. Respostas cardiorrespiratórias em exercícios de hidroginástica executados com e sem o uso de equipamento resistivo. Rev Port Cienc Desp 2006;6:336-41

17. Parker SB, Huerley BF, Hanlon DP, Vaccaro P. Failure of target heart rate to accurately monitor intensity during aerobic dance. Med Sci Sports Exerc 1989;21:230-4.

18. Martinovic NVP, Marques MB, Novaes JS. Respostas cardiovasculares e metabólicas do step training em diferentes alturas de plataforma. Rev Bras Ativ Física Saúde 2002;7:5-13.

19. Calegare AJA. Introdução ao delineamento de experimentos. São Paulo: Ed Edgard Blücher, 2001

20. Cooke CB. Metabolic rate and energy balance. In: Eston R, Reilly T, editors. Kinanthropometry and exercise physiology laboratory manual. London: E \& FN Spon 1996;175-95.

21. Linnarsson D. Dynamics of pulmonary gas exchange and heart rate changes at start and end of exercise. Acta Physiol Scand Suppl 1974;415:1-68.

22. Gleim GW, Nicholas JA. Metabolic costs and heart rate responses to treadmill walking in water at different depths and temperatures. Am J Sports Med 1989;17:248-52.

23. Müller FG, Alberton $C L$, Tartaruga LAP, Kruel LFM. Freqüência cardíaca em homens imersos em diferentes temperaturas de água. Rev Port Cienc Desp 2005;5:266-73.

24. Mcardle WD, Magel JR, Lesmes GR, Pechar GS. Metabolic and cardiovascular adjustment to work in air and water at 18,25 and $33^{\circ} \mathrm{C}$. J Appl Physiol 1976;40:85-90

25. Shono T, Fujishima K, Hotta N, Ogaki T, Ueda T, Otoki K, et al. Physiological responses and RPE during underwater treadmill walking in women of middle and advanced age. J Physiol Anthropol 2000;19:195-200.

26. Lagally KM, Robertson RJ, Gallagher KI, Goss FL, Jakicic JM, Lephart SM, et al. Perceived exertion, electromyography, and blood lactate during acute bouts of resistance exercise. Med Sci Sports Exerc 2002;34:552-9.

27. Doherty M, Smith PM, Hughes MG, Collins D. Rating of perceived exertion during high- intensity treadmill running. Med Sci Sports Exerc 2001;33:1953-8.

28. Gearhart RF, Goss FL, Lagally KM, Jakicic JM, Gallagher J, Gallagher Kl, et al. Ratings of perceived exertion in active muscle during high intensity and low intensity resistance exercise. J Strength Cond Res 2002;16:87-91.

29. Svedenhag J, Seger J. Running on land and in water: comparative exercise physiology. Med Sci Sports Exerc 1992;24:1155-60. 